Pacific Journal of Mathematics

INVARIANTS FOR 3-MANIFOLDS FROM THE
COMBINATORICS OF THE JONES POLYNOMIAL 


\title{
INVARIANTS FOR 3-MANIFOLDS FROM THE COMBINATORICS OF THE JONES POLYNOMIAL
}

\author{
W. B. R. LICKORISH
}

The bracket polynomial of Kauffman first gave an exceedingly simple definition of the Jones polynomial for links. Here it is used to give a short direct proof of the existence of a few of Witten's 3-manifold invariants.

The techniques of quantum field theory have been used by Witten [9] in the production of an array of invariants for 3-manifolds and for links in 3-manifolds. When the 3-manifold is the 3-sphere, these link invariants become the Jones polynomial (or one of its generalisations) evaluated at various complex roots of unity. A proof of the existence of such invariants has been given by Reshetikhin and Turaev [8] using deep results from the theory of quantum groups. An alternative approach, based on only the general outline of their method, is given here. This proof of the invariants' existence uses nothing but simple combinatorics and the well known theory of 3-manifolds being created by surgery on the 3-sphere. The result actually obtained here estiblishes only a very small selection of the new invariants, but the method has scope for extension (see however Appendix 2). The nature of the invariants is described in a fairly simple way, and those invariants that are here established are the only ones for which calculation seems to be at all feasible. Some of these calculations have been performed and discussed by Kirby and Melvin [4].

The basic tool that will be used is the bracket polynomial invariant of Kauffman [2], [7]. The bracket is a function

\langle\rangle$:\left\{\right.$ Diagrams in $\mathbf{R}^{2} \cup \infty$ of unoriented links $\} \rightarrow \mathbf{Z}\left[A^{ \pm 1}\right]$ that is defined by three properties:

(i) $\langle\varnothing\rangle=1$;

(ii) $\langle D \cup U\rangle=\delta\langle D\rangle$, where $U$ is a component with no crossing at all and $\delta=-A^{-2}-A^{2}$;

(iii) $\langle\backslash\rangle=A\langle\asymp\rangle+A^{-1}\langle\supset \subset\rangle$, where this refers to three diagrams identical except where shown.

(Note that the normalisation of (i) is not entirely standard.) It is 


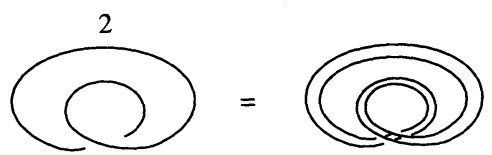

FIGURE 1
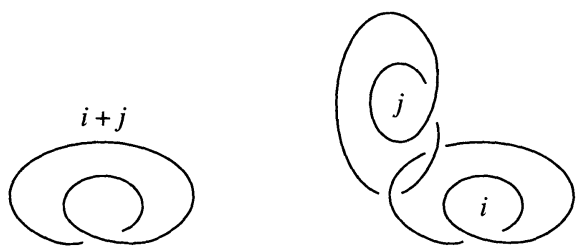

FIGURE 2

very easy to show (see [2] or [7]) that $\langle D\rangle$ is unchanged by both the second and third type of Reidemeister move (that is, it is a regular isotopy invariant). Its response to the first type of move is given by $\langle\supset\rangle=-A^{3}\langle\supset\rangle$. A further consequence of the definition is that, if $D_{1}$ and $D_{2}$ are disjoint diagrams, $\left\langle D_{1} \cup D_{2}\right\rangle=\left\langle D_{1}\right\rangle\left\langle D_{2}\right\rangle$. Now let $\tilde{w}(D)$ be the sum of the signs of all the crossings in $D$ where any component crosses itself; that is well defined without choosing any orientation on the components of $D$. If $L$ is a link in $S^{3}$ represented by $D$, define

$$
\widetilde{V}(L)=(-A)^{-3 \tilde{w}(D)}\langle D\rangle .
$$

This is easily seen to be invariant under all three types of Reidemeister move and so is an invariant of the unoriented link. For reference, if $L$ is given an orientation and if $\Lambda$ denotes the sum of the linking numbers between all pairs of components of $L$, then the standard Jones polynomial $V_{L}(t)$ is $\delta^{-1} A^{-6 \Lambda} \widetilde{V}(L)$, where $t=A^{-4}$. Thus $\widetilde{V}(L)$ is a disoriented Jones polynomial.

Various planar diagrams of links and parts of links will appear in what follows. A non-negative integer $i$ beside a component will signify the presence of $i$ copies of that component, all parallel in the plane. That is illustrated in Figure 1.

Figure 2 shows a pair of diagrams (of the Hopf link of $i+j$ components) which are regularly isotopic and so have the same bracket polynomial; let $T_{i+j}$ denote the bracket polynomial of either of these diagrams. The bracket takes values in the Laurent polynomial ring $\mathbf{Z}\left[A^{ \pm 1}\right]$, but in what follows it will be evaluated when $A$ is a specific root of unity, so that the bracket may be thought of as having complex number values.

It is well known [5] that any closed oriented 3-manifold $M^{3}$ can be obtained from the 3-sphere $S^{3}$ by surgery on a framed (unoriented) link $(L, f)$. Thus, to each component $L_{s}$ of $L$ is assigned a "framing" which is an integer $f(s) . M^{3}$ can be constructed by the 

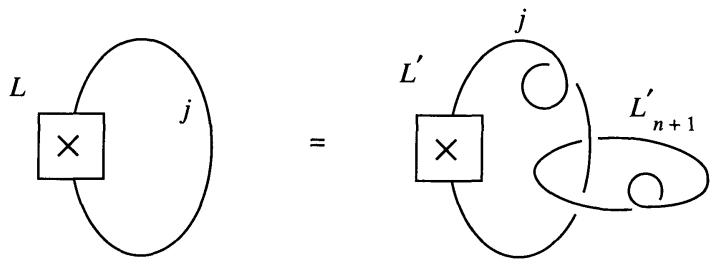

FIGURE 3

following process. Remove a small open solid torus neighbourhood of each $L_{s}$. On each resulting toral boundary component consider the simple closed curve that represents $f(s)$ meridians and one longitude of $L_{s}$; attach new solid tori so that each of these (framing) curves now bounds a disc. A theorem of Kirby [3], refined by Fenn and Rourke [1], asserts that framed links $(L, f)$ and $\left(L^{\prime}, f^{\prime}\right)$ give the same 3-manifold if they are related by ambient isotopy and by the equivalence relation generated by moves of two kinds. In the first, depicted in Figure 3, $\left(L^{\prime}, f^{\prime}\right)$ is obtained from $(L, f)$ by inserting an extra unknotted component $L_{n+1}^{\prime}$ with $f^{\prime}(n+1)=1$, adding a full positive twist in the strands linked by this component and defining $f^{\prime}(s)=f(s)+\left(\Lambda_{s}\right)^{2}$, where $\Lambda_{s}$ is the linking number of $L_{s}^{\prime}$ with the new component.

In the second kind of move, $\left(L^{\prime}, f^{\prime}\right)$ is $(L, f)$ together with an extra component, of framing -1 , that is unknotted and unlinked from $L$. (The moves of [1] include a "negative" version of the first move; a proof of the above simplification, due to Turaev, is shown in an appendix.) Employing this result, a 3-manifold invariant comes at once from any quantity associated to framed links in $S^{3}$ that is invariant under ambient isotopy and under the two types of move described above.

It is convenient to explain some notation before stating the theorem. If $(L, f)$ is a framed link with components $L_{1}, L_{2}, \ldots, L_{n}$, and $c$ is a function, $c:\{1,2, \ldots, n\} \rightarrow \mathbf{Z}_{+}$, let $c_{f}^{*} L$ be the link in which each component $L_{s}$ of $L$ has been replaced by $c(s)$ components all lying in the torus boundary of a small regular neighbourhood of $L_{s}$, all parallel to the framing curve. Usually $c$ will be restricted to $C(n, r)$, the set of all functions $c:\{1,2, \ldots, n\} \rightarrow\{0,1, \ldots, r-2\}$. If the framed link $(L, f)$ is given an orientation, the linking numbers of the pairs of its components form a symmetric matrix in which $f(s)$ is taken to be the linking number of $L_{s}$ with itself. The signature and nullity of this matrix are independent of the choice of orientations. The nullity 
of the matrix is, in fact, the first Betti number of the 3-manifold obtained by surgery along $(L, f)$. Recall that $T_{i+j}$ is the bracket of the diagram shown twice in Figure 2, and that $\delta=-A^{-2}-A^{2}$.

The following theorem is, then, a version of part of the results of Witten [9] as interpreted by Reshetikhin and Turaev. For an integer $r$ it produces an invariant of 3-manifolds in the complex numbers. The proof given here is very elementary. That is intended to be its virtue; its drawback is that it only works with ease for $r \leq 6$. Solution of an entirely combinatorial conjecture would extend the proof to all higher integers.

Theorem. Let $r \in\{3,4,5,6\}$ and let $A=e^{\pi i / 2 r}$. Let $\lambda_{0}, \lambda_{1}, \ldots$, $\lambda_{r-2}$ be a solution in the complex numbers to the linear equations

$$
\sum_{i=0}^{r-2} \lambda_{i} T_{i+j}=\delta^{j}, \quad j=0,1, \ldots, r-2 .
$$

Let $\kappa=\sum_{i=0}^{r-2} \lambda_{i} \bar{T}_{i}$. Suppose that $M^{3}$ is obtained from $S^{3}$ by surgery on an $n$-component framed link $(L, f)$, for which $\sigma$ and $\nu$ are the signature and nullity of the linking matrix. Then the expression

$$
\kappa^{(\sigma+\nu-n) / 2} \sum_{c \in C(n, r)} \lambda_{c(1)} \lambda_{c(2)} \cdots \lambda_{c(n)}(-A)^{3} \sum_{s=1}^{n} c(s) f(s) \widetilde{V}\left(c *_{f} L\right)
$$

is an invariant $I_{r}\left(M^{3}\right)$ of the 3-manifold, a complex number independent of the choice of $(L, f)$.

Notice that, in calculating $I_{r}\left(M^{3}\right)$ as described in this theorem, it is necessary to calculate $\widetilde{V}\left(c *_{f} L\right)$. If $r=6$ and $L$ is just the 3crossing knot, a diagram of four parallel copies of the knot has at least 48 crossings; naive calculation of the bracket polynomial (direct from its definition) would then involve $2^{48}$ operations.

The theorem's proof will use a simple version of linear skein theory (see [6]) that will now be described. Consider a square in $\mathbf{R}^{2} \cup \infty$ with $j$ specified points on its top edge and $j$ such points on its bottom edge. Consider all tangle diagrams in the square (i.e. link diagrams in which components may be arcs) with the specified points as boundary. Figure 4 shows an example when $j=3$.

Let $V_{j}^{\text {in }}$ be the module over $\mathbf{Z}\left[A^{ \pm 1}\right]$ freely generated by all such diagrams quotiented by relations of the form

(i) $D \cup U=\delta D$,

(ii) $(\searrow)=A(\asymp)+A^{-1}(\supset \subset)$. 


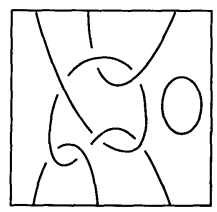

FIGURE 4

As before, in (i) $U$ is a closed component of the diagram that contains no crossing (one such is in Figure 4), and in (ii) the diagrams in parentheses are the same except where shown. Of course, equalities in this module are thought of as partial calculations of bracket polynomials, and, if a complex number is substituted for $A, V_{j}^{\text {in }}$ becomes a vector space. Let $V_{j}^{\text {out }}$ be defined in exactly the same way using diagrams outside the square. The operation, of placing one diagram inside the square, another outside the square, and taking the bracket polynomial of the resulting link diagram, extends to a well-defined bilinear form

$$
\langle,\rangle: V_{j}^{\text {out }} \times V_{j}^{\text {in }} \rightarrow \mathbf{Z}\left[A^{ \pm 1}\right] \text {. }
$$

Now, $V_{j}^{\text {in }}$ is freely generated by a base of all diagrams in the square with no crossing and no closed component. Thus the dimension of $V_{j}^{\text {in }}$ is the Catalan number $\frac{1}{j+1}\left(\begin{array}{c}2 j \\ j\end{array}\right)$. Figure 5 shows the base of $V_{3}^{\text {in }}$, the analogous base of $V_{3}^{\text {out }}$ and the resulting (symmetric) matrix for the above bilinear form. Let $u_{j}, \varepsilon_{j}$, and $\mu_{i, j}$ be the elements of $V_{j}^{\text {in }}$ and $V_{j}^{\text {out }}$ shown in Figure 6.

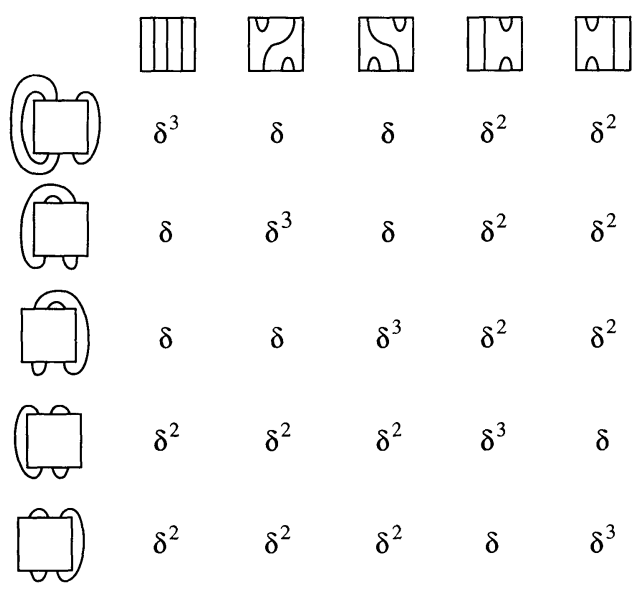

FIGURE 5 

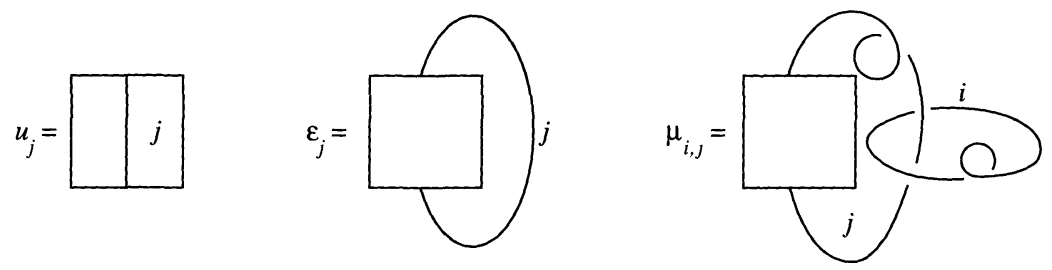

FIGURE 6

LEMMA 1. Let $\varphi_{j}=\sum_{i=0}^{r-2} \lambda_{i} \mu_{i, j} \in V_{j}^{\text {out }}$ where $\lambda_{0}, \lambda_{1}, \ldots, \lambda_{r-2}$ are as in the statement of the theorem. Then $\left\langle\varphi_{j},\right\rangle$ and $\left\langle\varepsilon_{j},\right\rangle$ are equal linear maps of $V_{j}^{\text {in }}$ for $j=0,1, \ldots, r-2$.

Proof. The definition of $\left\{\lambda_{i}\right\}$ means that

$$
\left\langle\varphi_{j}, u_{j}\right\rangle=\delta^{j}=\left\langle\varepsilon_{j}, u_{j}\right\rangle, \quad \text { for } j=0,1, \ldots, r-2 .
$$

Suppose that $e$ is any base element of $V_{j}^{\text {in }}$ other than $u_{j}$. Consider the annulus, shown in Figure 7, containing a square with $j$ parallel arcs joining the top and bottom of the square as shown.

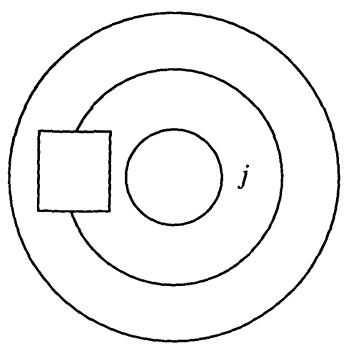

Figure 7

Inserting $e$ into this square would produce a configuration of disjoint simple closed curves. These may be isotoped in the annulus to $x$, say, standard mutually parallel curves encircling the annulus and $y$ small nul-homotopic curves (which may be nested). Note that $x<j$. Then

$$
\left\langle\mu_{i, j}, e\right\rangle=\delta^{y}\left\langle\mu_{i, x}, u_{x}\right\rangle \text {. }
$$

This is because the isotopy in the above annulus induces a regular isotopy of the diagram consisting of $e$ in the square of $\mu_{i, j}$. The end of the regular isotopy is $u_{x}$ in $\mu_{i, x}$, together with $y$ components with no crossing. Then

$$
\left\langle\varphi_{j}, e\right\rangle=\delta^{y}\left\langle\varphi_{x}, u_{x}\right\rangle=\delta^{x+y}
$$


However, $\left\langle\varepsilon_{j}, e\right\rangle=\delta^{x+y}$, so the result now follows since $\left\langle\varphi_{j},\right\rangle$ and $\left\langle\varepsilon_{j},\right\rangle$ agree on a base of $V_{j}^{\text {in }}$.

Now, for $r \in\{2,3,4,5,6\}$ inspection shows that when $A=$ $e^{\pi i / 2 r}$ the bilinear form

$$
\langle,\rangle: V_{r-1}^{\text {out }} \times V_{r-1}^{\text {in }} \rightarrow \mathbf{C}
$$

is degenerate, and that $\left\langle, u_{r-1}\right\rangle$ can be expressed as a linear combination of the $\left\langle, g_{k}\right\rangle$ where the $g_{k}$ are the base elements of $V_{r-1}^{\text {in }}$ other than $u_{r-1}$. For example, consider the case when $r=4$. Then $A=e^{\pi i / 8}$ and $\delta=-\sqrt{2}$. The matrix of Figure 5 is singular, and $(1,1,1, \sqrt{2}, \sqrt{2})$ is an eigenvector with zero eigenvalue. If $j>r-1$ it will then also be true that $\left\langle, u_{j}\right\rangle=\sum_{k}\left\langle, \alpha_{k} e_{k}\right\rangle$ for some $\alpha_{k} \in \mathbf{C}$, where the $e_{k}$ are the base elements of $V_{j}^{\text {in }}$ other than $u_{j}$. This is because the $j$ arcs of $u_{j}$ can be considered as $r-1$ arcs beside $j-r+1$ arcs; if $\left\langle, u_{r-1}\right\rangle=\sum_{k}\left\langle, \beta_{k} g_{k}\right\rangle$ then $\left\langle, u_{j}\right\rangle=\sum_{k}\left\langle, \beta_{k} g_{k}^{+}\right\rangle$where $g_{k}^{+}$is the diagram $g_{k}$ with $j-r+1$ extra vertical arcs.

LEMMA 2. Suppose that $\left\langle, u_{r-1}\right\rangle$ can be expressed as a linear combination of the $\left\langle, g_{k}\right\rangle$ where the $g_{k}$ are the base elements of $V_{r-1}^{\text {in }}$ other than $u_{r-1}$. Then $\left\langle\varphi_{j},\right\rangle=\left\langle\varepsilon_{j},\right\rangle$ for all $j=0,1,2, \ldots$.

Proof. Suppose inductively that the lemma has been proved for all integers less than $j$; Lemma 1 starts this induction. If $j \geq r-1$, then

$$
\left\langle, u_{j}\right\rangle=\sum_{k}\left\langle, \alpha_{k} e_{k}\right\rangle
$$

as explained above, the $e_{k}$ being base elements of $V_{j}^{\text {in }}$ other than $u_{j}$. As before, $e_{k}$ when inserted into the diagram of Figure 7 produces $x(k)$ essential curves, $x(k)<j$, and $y(k)$ inessential curves. Then

$$
\begin{aligned}
\left\langle\varphi_{j}, u_{j}\right\rangle & =\sum_{k} \alpha_{k}\left\langle\varphi_{j}, e_{k}\right\rangle=\sum_{k} \alpha_{k} \delta^{y(k)}\left\langle\varphi_{x(k)}, u_{x(k)}\right\rangle \\
& =\sum_{k} \alpha_{k} \delta^{y(k)}\left\langle\varepsilon_{x(k)}, u_{x(k)}\right\rangle \quad \text { (by the induction) } \\
& =\sum_{k} \alpha_{k} \delta^{y(k)+x(k)} \\
& =\sum_{k} \alpha_{k}\left\langle\varepsilon_{j}, e_{k}\right\rangle=\left\langle\varepsilon_{j}, u_{j}\right\rangle .
\end{aligned}
$$

Thus $\left\langle\varphi_{k}, u_{k}\right\rangle=\left\langle\varepsilon_{k}, u_{k}\right\rangle$ for $k=0,1, \ldots, j$ and so the proof of Lemma 1 implies that $\left\langle\varphi_{j},\right\rangle=\left\langle\varepsilon_{j},\right\rangle$. 
Proof of the Theorem. Suppose that $(L, f)$ and $\left(L^{\prime}, f^{\prime}\right)$ are framed links with diagrams related as in Figure $3, L$ having $n$ components, where $f^{\prime}(n+1)=1$, and $f^{\prime}(s)=f(s)+\left(\Lambda_{s}\right)^{2}$ for $s \leq n \quad\left(\Lambda_{s}\right.$ being the linking number between $L_{s}^{\prime}$ and $\left.L_{n+1}^{\prime}\right)$. If $c \in C(n, r)$, let $c_{i}^{\prime} \in$ $C(n+1, r)$ be defined by $c_{i}^{\prime}(s)=c(s)$ for $s \leq n$, and $c_{i}^{\prime}(n+1)=i$. Let $D$ and $D_{i}^{\prime}$ be diagrams of $c *_{f} L$ and $c_{i}^{\prime} *_{f^{\prime}} L^{\prime}$ that are the same inside the squares of Figure 3 and outside consist of curves parallel in the plane to those shown. Then $\tilde{w}\left(D_{i}^{\prime}\right)=\tilde{w}(D)+i+\sum_{s=1}^{n} c(s) \Lambda_{s}^{2}$. From Lemma 2

$$
\sum_{i=0}^{r-2} \lambda_{i}\left\langle D_{i}^{\prime}\right\rangle=\langle D\rangle
$$

But $\widetilde{V}\left(c *_{f} L\right)=(-A)^{-3 \tilde{w}(D)}\langle D\rangle$ a similar expression holding for $c_{i}^{\prime} * f^{\prime} L^{\prime}$. Thus

$$
\sum_{i=0}^{r-2} \lambda_{i}(-A)^{3\left(i+\sum c(s) \Lambda_{s}^{2}\right)} \tilde{V}\left(c_{i}^{\prime} *_{f^{\prime}} L^{\prime}\right)=\tilde{V}\left(c *_{f} L\right)
$$

Multiplying this by $\lambda_{c(1)} \lambda_{c(2)} \cdots \lambda_{c(n)}(-A)^{3} \sum c(s) f(s)$ and adding gives

$$
\begin{gathered}
\sum_{c^{\prime} \in C(n+1, r)} \lambda_{c^{\prime}(1)} \lambda_{c^{\prime}(2)} \cdots \lambda_{c^{\prime}(n+1)}(-A)^{3} \sum_{s=1}^{n+1} c^{\prime}(s) f^{\prime}(s) \tilde{V}\left(c_{i}^{\prime} * f^{\prime} L^{\prime}\right) \\
=\sum_{c \in C(n, r)} \lambda_{c(1)} \lambda_{c(2)} \cdots \lambda_{c(n)}(-A)^{3} \sum_{s=1}^{n} c(s) f(s) \tilde{V}\left(c *_{f} L\right) .
\end{gathered}
$$

Thus, if $X(L, f)$ denotes the expression on the right of this equation, $X\left(L^{\prime}, f^{\prime}\right)=X(L, f)$.

It remains to consider the other basic move on framed link diagrams, namely when $(L, f)$ is changed to $\left(L^{\prime \prime}, f^{\prime \prime}\right)$, a new framed link that is equal to $(L, f)$ except for the insertion of an extra unknotted component, unlinked from the original components, and with framing -1 . However, if links $L_{1}$ and $L_{2}$ are separated by a 2-sphere in $\mathbf{R}^{3}$, then $\widetilde{V}\left(L_{1} \cup L_{2}\right)=\widetilde{V}\left(L_{1}\right) \widetilde{V}\left(L_{2}\right)$. Hence

$$
X\left(L^{\prime \prime}, f^{\prime \prime}\right)=X(L, f) \sum_{i=0}^{r-2} \lambda_{i}(-A)^{-3 i} \widetilde{V}\left(i *_{-1} U\right)
$$

where $i *_{-1} U$ is $i$ parallel copies of the unknot using the -1 framing When $A$ is a root of unity and $\bar{D}$ is the reflection of a link diagram $D$, then $\langle\bar{D}\rangle$ is the complex conjugate of $\langle D\rangle$. Hence $\widetilde{V}\left(i *_{-1} U\right)=$ $(-A)^{3 i} \bar{T}_{i}$ and $X\left(L^{\prime \prime}, f^{\prime \prime}\right)=\kappa X(L, f)$. But the $n \times n$ linking matrix of $L$ has signature $\sigma$ and nullity $\nu$, so $\frac{1}{2}(n-\sigma-\nu)$ is the number 
of negative entries in a diagonalisation of the matrix. That number is unchanged when $(L, f)$ is changed (as in the first move above) to $\left(L^{\prime}, f^{\prime}\right)$ and increases by one if the change is to $\left(L^{\prime \prime}, f^{\prime \prime}\right)$. Hence $\kappa^{(\sigma+\nu-n) / 2} X(L, f)$ is invariant under both types of move, and also, of course, under ambient isotopy of $L$.

The above ideas generalise at once to give invariants of framed links in the 3-manifold $M^{3}$ : Suppose that $K$ is a framed $m$-component link in $M^{3}$ where, as before, $M^{3}$ is obtained by surgery on the framed link $(L, f)$ in $S^{3}$. Then $K$ can be regarded as a link with framing $g$ in $S^{3}-L$. If $d:\{1,2, \ldots, m\} \rightarrow \mathbf{Z}_{+}$, an invariant of $(K, g, d)$ is given by the expression

$$
\begin{array}{r}
\kappa^{(\sigma+\nu-n) / 2} \sum_{c \in C(n, r)} \lambda_{c(1)} \lambda_{c(2)} \cdots \lambda_{c(n)}(-A)^{3} \sum_{s=1}^{n} c(s) f(s) \\
\times \widetilde{V}((c \cup d) * f \cup g(L \cup K)) .
\end{array}
$$

Of course, if $d$ has all values 1 and $M^{3}$ is $S^{3}$, this reduces to $\widetilde{V}(K)$ evaluated at $A=e^{i \pi / 2 r}$.

It is disappointing that this proof can, at present, claim the theorem only for $r \leq 6$. In the circumstances it seems reasonable to make the conjecture (but see Appendix 2) that, in the matrix representing $\langle$,$\rangle with respect to the standard bases of V_{r-1}^{\text {in }}$ and $V_{r-1}^{\text {out }}$ the column corresponding to $u_{r-1}$ is a linear combination of the other columns when $A=e^{i \pi / 2 r}$. This is a combinatorial problem, the matrix entries being of the form $\left(-A^{-2}-A^{2}\right)^{N}$ where $N$ is the number of simple closed curves that arise when one basic diagram is placed inside the square and the other on the outside (see Figure 5). When $r=6$ the matrix has $42^{2}$ entries but there is enough symmetry available to make manual calculation possible. For $r=7$ there are $132^{2}$ entries. Whenever the conjecture is correct, the above method shows $I_{r}\left(M^{3}\right)$ to be an invariant. Note that the matrix does, of course, have zero determinant for some value of $A$.

An explicit calculation of the $\lambda_{i}$ appears in [4], where an exploration is made of the possibility of interpreting $I_{r}\left(M^{3}\right)$ in terms of other, more classical, 3-manifold invariants. Such an interpretation is to be expected when $r=3,4$ or 6 , because there are interpretations, in terms of classical link invariants, of the Jones polynomial evaluated at a third, fourth or sixth root of unity. No such interpretation is available when $r=5$. 

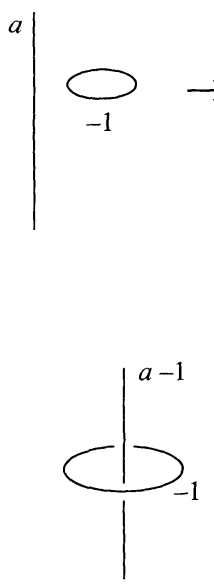

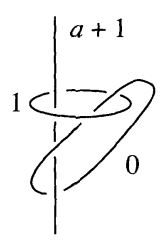

$\leftarrow$
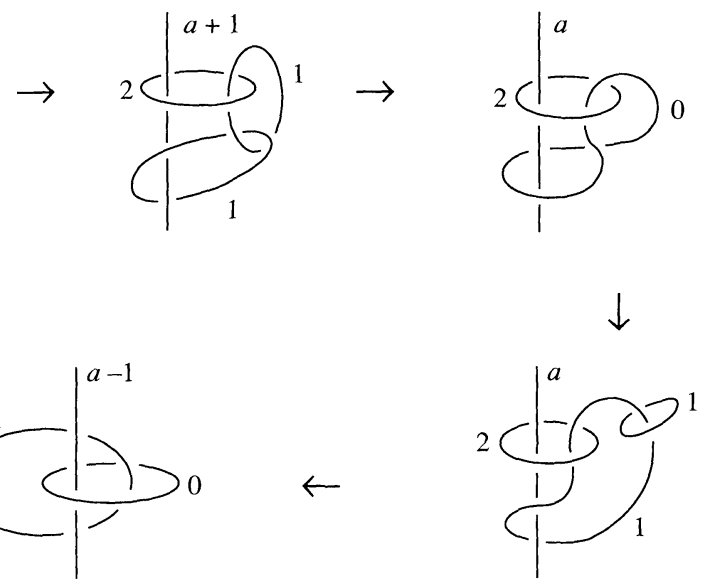

Figure 8

Appendix 1. The sequence of diagrams of Figure 8 shows that the two moves described above on framed links do indeed generate the negative version of the first type of move. Here the numbers denote framings.

Appendix 2. Subsequent to the writing of this paper, the conjecture that follows the proof of the theorem, concerning the degeneracy of $\langle$,$\rangle on V_{r-1}^{\text {out }} \times V_{r-1}^{\text {in }}$ when $A=e^{\pi i / 2 r}$, has been proved correct by K. H. Ko and L. Smolinsky. Their Louisiana State University preprint "A combinatorial matrix in 3-manifold theory" is to be published in this Journal. The proof consists of a carefully controlled argument concerning elementary row and column operations on the matrix representing the form. When $r \leq 6$ the existence of $\lambda_{0}, \lambda_{1}, \ldots, \lambda_{r-2}$ solving the linear equations mentioned in the statement of the theorem can be verified by checking the non-singularity of, at worst, a specific $5 \times 5$ matrix. For all values of $r$ this non-singularity can be proved using the additional information provided by Ko and Smolinsky that $\langle$,$\rangle is non-degenerate on V_{j}^{\text {out }} \times V_{j}^{\text {in }}$ for $j \leq(r-2)$. Details will appear elsewhere. Taken together, these papers become the basis of an elementary proof of the existence of a 3-manifold invariant, associated to the Jones polynomial, for every root of unity. It transpires that the solution to the above mentioned conjecture is inherent (though not explicitly stated) in the paper "Index of subfactors" by V. F. R. Jones (Invent. Math. 72 (1983), 1-25). 


\section{REFERENCES}

[1] R. A. Fenn and C. P. Rourke, On Kirby's calculus of links, Topology, 18 (1979), $1-15$.

[2] L. H. Kauffman, State models and the Jones polynomial, Topology, 26 (1987), 395-407.

[3] R. C. Kirby, A calculus for framed links in $S^{3}$, Invent. Math., 45 (1978), 35-56.

[4] R. C. Kirby and P. Melvin, Evaluations of the 3-manifold invariants of Witten and Reshetikhin-Turaev for $\mathbf{s l}(2, \mathbf{C})$, (to appear).

[5] W. B. R. Lickorish, A representation of orientable combinatorial 3-manifolds, Ann. of Math., 76 (1962), 531-540.

[6] Linear skein theory and link polynomials, Topology Appl., 27 (1987), 265-274.

[7] _ Polynomials for links, Bull. London Math. Soc., 20 (1988), 558-588.

[8] N. Y. Reshetikhin and V. G. Turaev, Invariants of 3-manifolds via link polynomials and quantum groups, to appear, Invent. Math.

[9] E. Witten, Quantum field theory and Jones' polynomial, Comm. Math. Phys., 121 (1989), 351-399.

Received February 1, 1990 and in revised form April 17, 1990.

UNIVERSITY OF CAMBRIDGE

16, Mill LaNe

CAmbridge, CB2 1SB, Great Britain 



\section{PACIFIC JOURNAL OF MATHEMATICS EDITORS}

\author{
V. S. VARAdaraJan \\ (Managing Editor) \\ University of California \\ Los Angeles, CA 90024-1555-05 \\ Herbert Clemens \\ University of Utah \\ Salt Lake City, UT 84112 \\ THOMAS ENRIGHT \\ University of California, San Diego \\ La Jolla, CA 92093
}

R. FINN

Stanford University

Stanford, CA 94305

Hermann FlaschKa

University of Arizona

Tucson, AZ 85721

VAUGHAN F. R. Jones

University of California

Berkeley, CA 94720

STEVEN KerckHofF

Stanford University

Stanford, CA 94305

\section{C. MOORE}

University of California

Berkeley, CA 94720

Martin ScharlemanN

University of California

Santa Barbara, CA 93106

HAROLd STARK

University of California, San Diego

La Jolla, CA 92093
R. ARENS
E. F. BECKENBACH
F. WoLF
K. YoshidA
ASSOCIATE EDITORS

\begin{tabular}{ll}
\multicolumn{1}{c}{$(1906-1982)$} & \multicolumn{1}{c}{$(1904-1989)$} \\
SUPPORTING & INSTITUTIONS \\
UNIVERSITY OF ARIZONA & UNIVERSITY OF OREGON \\
UNIVERSITY OF BRITISH COLUMBIA & UNIVERSITY OF SOUTHERN CALIFORNIA \\
CALIFORNIA INSTITUTE OF TECHNOLOGY & STANFORD UNIVERSITY \\
UNIVERSITY OF CALIFORNIA & UNIVERSITY OF HAWAII \\
MONTANA STATE UNIVERSITY & UNIVERSITY OF TOKYO \\
UNIVERSITY OF NEVADA, RENO & UNIVERSITY OF UTAH \\
NEW MEXICO STATE UNIVERSITY & WASHINGTON STATE UNIVERSITY \\
OREGON STATE UNIVERSITY & UNIVERSITY OF WASHINGTON
\end{tabular}

The Supporting Institutions listed above contribute to the cost of publication of this Journal, but they are not owners or publishers and have no responsibility for its content or policies.

Mathematical papers intended for publication in the Pacific Journal of Mathematics should be in typed form or offset-reproduced (not dittoed), double spaced with large margins. Please do not use built up fractions in the text of the manuscript. However, you may use them in the displayed equations. Underline Greek letters in red, German in green, and script in blue. The first paragraph must be capable of being used separately as a synopsis of the entire paper. In particular it should contain no bibliographic references. Please propose a heading for the odd numbered pages of less than 35 characters. Manuscripts, in triplicate, may be sent to any one of the editors. Please classify according to the 1991 Mathematics Subject Classification scheme which can be found in the December index volumes of Mathematical Reviews. Supply name and address of author to whom proofs should be sent. All other communications should be addressed to the managing editor, or Elaine Barth, University of California, Los Angeles, California 90024-1555-05.

There are page-charges associated with articles appearing in the Pacific Journal of Mathematics. These charges are expected to be paid by the author's University, Government Agency or Company. If the author or authors do not have access to such Institutional support these charges are waived. Single authors will receive 50 free reprints; joint authors will receive a total of 100 free reprints. Additional copies may be obtained at cost in multiples of 50 .

The Pacific Journal of Mathematics (ISSN 0030-8730) is published monthly except for July and August. Regular subscription rate: $\$ 190.00$ a year (10 issues). Special rate: $\$ 95.00$ a year to individual members of supporting institutions.

Subscriptions, orders for numbers issued in the last three calendar years, and changes of address should be sent to Pacific Journal of Mathematics, P.O. Box 969, Carmel Valley, CA 93924, U.S.A. Old back numbers obtainable from Kraus Periodicals Co., Route 100, Millwood, NY 10546.

The Pacific Journal of Mathematics at P.O. Box 969, Carmel Valley, CA 93924 (ISSN 0030-8730) is published monthly except for July and August. Second-class postage paid at Carmel Valley, California 93924, and additional mailing offices. Postmaster: send address changes to Pacific Journal of Mathematics, P.O. Box 969, Carmel Valley, CA 93924.

PUBLISHED BY PACIFIC JOURNAL OF MATHEMATICS, A NON-PROFIT CORPORATION Copyright (C) 1991 by Pacific Journal of Mathematics 


\section{Pacific Journal of Mathematics}

Vol. 149, No. $2 \quad$ June, 1991

Manuel Alfaro Garcia, Mark Conger and Kenneth Hodges, The structure

of singularities in $\Phi$-minimizing networks in $\mathbf{R}^{2}$

Werner Balser, Dependence of differential equations upon parameters in

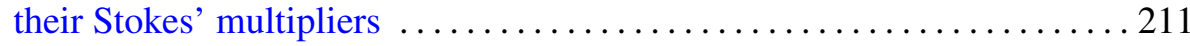

Enrico Casadio Tarabusi and Stefano Trapani, Envelopes of holomorphy

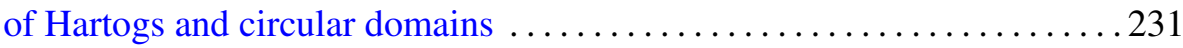

Hermann Flaschka and Luc Haine, Torus orbits in $G / P \ldots \ldots \ldots \ldots 251$

Gyo Taek Jin, The Cochran sequences of semi-boundary links .......... 293

Yasuyuki Kawahigashi, Cohomology of actions of discrete groups on

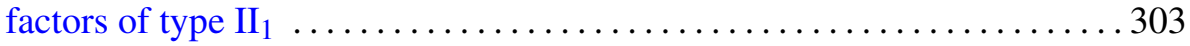

Ki Hyoung Ko and Lawrence Smolinsky, A combinatorial matrix in 3-manifold theory

W. B. Raymond Lickorish, Invariants for 3-manifolds from the combinatorics of the Jones polynomial

Peter Arnold Linnell, Zero divisors and group von Neumann algebras . . . . . 349

Bruce Harvey Wagner, Classification of essential commutants of abelian

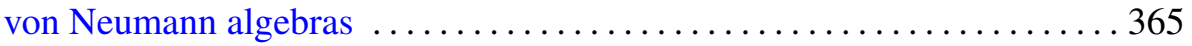

Herbert Walum, Multiplication formulae for periodic functions ..........383 